\title{
Closed-loop Subspace Identification with Innovation Estimation
}

\author{
S. Joe Qin, Lennart Ljung \\ Division of Automatic Control \\ Department of Electrical Engineering \\ Linköpings universitet, SE-581 83 Linköping, Sweden \\ WWW: http://www. control.isy.liu.se \\ E-mail: ljung@isy.liu.se, @isy.liu.se
}

3rd December 2003

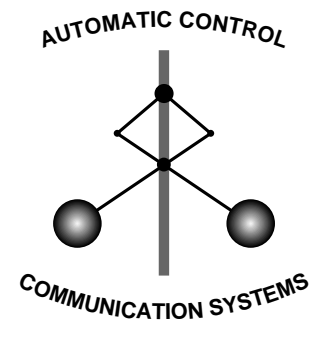

LINKÖPING

Report no.: LiTH-ISY-R-2559

Submitted to The 13th IFAC Symposium on System Identification, Rotterdam, The Netherlands

Technical reports from the Control \& Communication group in Linköping are available at http://www. control.isy.liu.se/publications. 


\title{
CLOSED-LOOP SUBSPACE IDENTIFICATION WITH INNOVATION ESTIMATION
}

\author{
S. Joe Qin* and Lennart Ljung ** \\ * Department of Chemical Engineering \\ The University of Texas at Austin \\ Austin, TX 78712, USA \\ e-mail: qin@che.utexas.edu. \\ ** Department of Electrical Engineering \\ Linkoping University \\ Linkoping, Sweden
}

\begin{abstract}
Most subspace identification algorithms are not applicable to closedloop identification because they require future input to be uncorrelated with past innovation. In this paper, we propose a new subspace identification method that remove this requirement by using a parsimonious model formulation with innovation estimation. A simulation example is included to show the effectiveness of the proposed method.
\end{abstract}

Keywords: subspace identification; closed-loop identification; parsimonious models; innovation estimation

\section{INTRODUCTION}

Subspace identification methods (SIM) have gone through tremendous development over the last decade (Chou and Verhaegen, 1997; Moor et al., 1988; Larimore, 1990; Moonen et al., 1989; Overschee and Moor, 1993; Overschee and Moor, 1994; Verhaegen, 1991; Verhaegen, 1994; Viberg, 1994). Among these algorithms canonical variate analysis (CVA)(Larimore, 1983; Larimore, 1990), N4SID (Overschee and Moor, 1994), MOESP (Verhaegen and Dewilde, 1992), and IV-4SID (Viberg, 1995) are some of the representing algorithms. These SIM algorithms are now well explained in many papers and several books (Overschee and Moor, 1996), (Ljung, 1999). Typically, an SIM estimates the extended observability matrix with or without estimating the state sequence. Then the system matrices and the disturbance characteristics are estimated. The unifying theorem (Overschee and de Moor, 1995; Jansson and Wahlberg, 1998) formulates many of the SIM algorithms in a singular value decomposition framework, with differences in weighting matrices. The CVA and MOESP weighting matrices have been proven to be approximately optimal weighting (Gustafsson, 2002). In addition, statistical properties such as consistency have recently been explored (Bauer et al., 1999; Deistler et al., 1995; Heij and Scherrer, 1999; Jansson and Wahlberg, 1998).

Although the SIM algorithms are attractive because the state space form is very convenient for estimation, filtering, prediction of multivariable systems, severe drawbacks have been experienced. In general, the SIM estimates are not as accurate as the prediction error methods (PEM). Further, very few, if any, SIM methods are applicable to closed-loop identification, even though the data satisfy identifiability conditions for traditional methods such as PEMs.

In a companion paper (Qin and Ljung, 2003), we give the reasons why subspace identification approaches exhibit these drawbacks and propose parallel parsimonious SIM (PARSIM-P) for open- 
loop applications. With the analysis of existing subspace formulation using the linear regression formulation (Jansson and Wahlberg, 1996; Jansson and Wahlberg, 1998; Knudsen, 2001), we reveal that the typical SIM algorithms actually use non-parsimonious model formulation, with extra terms in the model that appear to be non-causal. These terms, although conveniently included for performing subspace projection, are the causes for inflated variance in the estimates and partially responsible for the loss of closed-loop identifiability.

Removing the non-causal terms in the SIM formulation makes the model parsimonious, but it does not make the SIM methods automatically applicable to closed-loop identification. As pointed out in (Qin and Ljung, 2003), the PARSIM-P algorithm still requires that there is no correlation between future input $u_{k}$ and past innovation $e_{k}$, which is not the case for closed-loop data.

In this paper, we propose a new parsimonious method that removes this requirement, thus making it applicable to closed-loop identification. We propose to estimate the innovation process $e_{k}$ using the intermediate results in SIMs. Then the estimated innovation sequence is used in the subsequent projections in the SIM procedure. This method will be referred to as PARSIM-E, which means that the innovation process $e_{k}$ is estimated first. A simulation example is used to demonstrate the effectiveness of the proposed PARSIME method for closed-loop identification with comparison to the PARSIM-P and MOESP algorithms.

\section{SUBSPACE MODEL}

\subsection{Conventional Subspace Models}

We begin with an innovation model formulation,

$$
\begin{aligned}
x_{k+1} & =A x_{k}+B u_{k}+K e_{k} \\
y_{k} & =C x_{k}+D u_{k}+e_{k}
\end{aligned}
$$

where $y_{k} \in R^{n_{y}}, x_{k} \in R^{n}, u_{k} \in R^{n_{u}}$, and $e_{k} \in R^{n_{y}}$ are the system output, state, input, and innovation, respectively. $A, B, C, D$ and $K$ are system matrices with appropriate dimensions.

An extended state space model can be formulated as

$$
\begin{aligned}
& Y_{f}=\Gamma_{f} X_{k}+H_{f} U_{f}+G_{f} E_{f} \\
& Y_{p}=\Gamma_{p} X_{k-p}+H_{p} U_{p}+G_{p} E_{p}
\end{aligned}
$$

where the extended observability matrix

$$
\Gamma_{f}=\left[\begin{array}{c}
C \\
C A \\
\vdots \\
C A^{f-1}
\end{array}\right]
$$

and the Toeplitz matrices are

$$
\begin{aligned}
H_{f}= & {\left[\begin{array}{cccc}
D & 0 & \cdots & 0 \\
C B & D & \cdots & 0 \\
\vdots & \vdots & \ddots & \vdots \\
C A^{f-2} B & C A^{f-3} B & \cdots & D
\end{array}\right] } \\
G_{f}= & {\left[\begin{array}{cccc}
I & 0 & \cdots & 0 \\
C K & I & \cdots & 0 \\
\vdots & \vdots & \ddots & \vdots \\
C A^{f-2} K & C A^{f-3} K & \cdots & I
\end{array}\right] }
\end{aligned}
$$

The input and output data are arranged in the following Hankel form:

$$
\begin{aligned}
U_{f} & =\left[\begin{array}{cccc}
u_{k} & u_{k+1} & \cdots & u_{k+N-1} \\
u_{k+1} & u_{k+2} & \cdots & u_{k+N} \\
\vdots & \vdots & \ddots & \vdots \\
u_{k+f-1} & u_{k+f} & \cdots & u_{k+f+N-2}
\end{array}\right] \\
& \triangleq\left[\begin{array}{llll}
u_{f}(k) & u_{f}(k+1) & \cdots & u_{f}(k+N-1)
\end{array}\right] \\
U_{p} & =\left[\begin{array}{cccc}
u_{k-p} & u_{k-p+1} & \cdots & u_{k-p+N-1} \\
u_{k-p+1} & u_{k-p+2} & \cdots & u_{k-p+N} \\
\vdots & \vdots & \ddots & \vdots \\
u_{k-1} & u_{k} & \cdots & u_{k+N-2}
\end{array}\right] \\
& \triangleq\left[u_{p}(k-p) u_{p}(k-p+1) \cdots u_{p}(k-p+N-1)\right]
\end{aligned}
$$

Denoting

$$
\begin{gathered}
L^{1}=\left[\begin{array}{cccc}
L_{11}^{1} & L_{12}^{1} & \cdots & L_{1 p}^{1} \\
L_{21}^{1} & L_{22}^{1} & \cdots & L_{2 p}^{1} \\
\vdots & & \ddots & \\
L_{f 1}^{1} & L_{f 1}^{1} & & L_{f p}^{1}
\end{array}\right] \triangleq\left[\begin{array}{c}
L_{1}^{1} \\
L_{2}^{1} \\
\vdots \\
L_{f}^{1}
\end{array}\right] \\
L^{2}=\left[\begin{array}{cccc}
L_{11}^{2} & L_{12}^{2} & \cdots & L_{1 p}^{2} \\
L_{21}^{2} & L_{22}^{2} & \cdots & L_{2 p}^{2} \\
\vdots & & \ddots & \\
L_{f 1}^{2} & L_{f 1}^{2} & & L_{f p}^{2}
\end{array}\right] \triangleq\left[\begin{array}{c}
L_{1}^{2} \\
L_{2}^{2} \\
\vdots \\
L_{f}^{2}
\end{array}\right] \\
L^{3}=\left[\begin{array}{cccc}
L_{11}^{3} & L_{12}^{3} & \cdots & L_{1 f}^{3} \\
L_{21}^{3} & L_{22}^{3} & \cdots & L_{2 f}^{3} \\
\vdots & & \ddots & \\
L_{f 1}^{3} & L_{f 1}^{3} & & L_{f f}^{3}
\end{array}\right] \triangleq\left[\begin{array}{c}
L_{1}^{3} \\
L_{2}^{3} \\
\vdots \\
L_{f}^{3}
\end{array}\right]
\end{gathered}
$$

the above problem is equivalent to $f$ separate subproblems:

$$
\left[\begin{array}{lll}
\hat{L}_{i}^{1} & \hat{L}_{i}^{2} & \hat{L}_{i}^{3}
\end{array}\right]=\arg \min \{J\}
$$

where 


$$
\begin{aligned}
& J=\sum_{j=0}^{N-1}\left\|y(k+j+i-1)-\left[\begin{array}{lll}
L_{i}^{1} & L_{i}^{2} & L_{i}^{3}
\end{array}\right]\left[\begin{array}{c}
y_{p}(k-p+j) \\
u_{p}(k-p+j) \\
u_{f}(k+j)
\end{array}\right]\right\|^{2} \\
& \quad \text { for } i=1,2, \ldots, f
\end{aligned}
$$

For the case of $i=1$, for example, the problem implies that the following model is specified:

$$
\begin{array}{r}
y(k)=\left[\begin{array}{lll}
L_{1}^{1} & L_{1}^{2} & L_{1}^{3}
\end{array}\right]\left[\begin{array}{c}
y_{p}(k-p) \\
u_{p}(k-p) \\
u_{f}(k)
\end{array}\right]+v(k) \\
=\left[\begin{array}{ll}
L_{1}^{1} & L_{1}^{2}
\end{array}\right]\left[\begin{array}{c}
y_{p}(k-p) \\
u_{p}(k-p)
\end{array}\right]+L_{11}^{3} u(k) \\
+\sum_{j=2}^{f} L_{1 j}^{3} u(k+j-1)+v(k)
\end{array}
$$

Note that the third term on the RHS of the above equation is non-causal and unnecessary. Therefore, the model format used in SIM during the projection step is non-causal. This would result in non-causal models in the projection step. Although the non-causal terms are ignored at the step to estimate $B, D$, all the model parameters estimate have inflated variance due to the fact that extra and unnecessary terms are included in the model, making the model non-parsimonious. For $i>1$ the number of non-causal terms will reduce, but they are unnecessary as long as $i<f$.

To avoid these problems the SIM model must not include these non-causal terms. The PARSIM$\mathrm{P}$ algorithms remove: these terms by enforcing triangular structure of the Toeplitz matrix $H_{f}$ at every step of the SIM procedure. The approach are referred to as parsimonious subspace identification methods (PARSIM) as it uses parsimonious model formulation.

\subsection{Parsimonious Subspace Models}

The key idea in the proposed method is to exclude those non-causal terms of $U_{f}$. To accomplish this we partition the extended state space model rowwise as follows:

$$
Y_{f}=\left[\begin{array}{c}
Y_{f 1} \\
Y_{f 2} \\
\vdots \\
Y_{f f}
\end{array}\right] ; Y_{i} \triangleq\left[\begin{array}{c}
Y_{f 1} \\
Y_{f 2} \\
\vdots \\
Y_{f i}
\end{array}\right] ; i=1,2, \ldots, f
$$

Partition $U_{f}$ and $E_{f}$ in a similar way to define $U_{f i}$, $U_{i}, E_{f i}$, and $E_{i}$, respectively, for $i=1,2, \ldots, f$. Denote further

$$
\begin{aligned}
\Gamma_{f} & =\left[\begin{array}{c}
\Gamma_{f 1} \\
\Gamma_{f 2} \\
\vdots \\
\Gamma_{f f}
\end{array}\right] \\
H_{f i} & \triangleq\left[\begin{array}{llll}
C A^{i-2} B & \cdots & C B & D
\end{array}\right] \\
& \triangleq\left[\begin{array}{llll}
H_{i-1} & \cdots & H_{1} & H_{0}
\end{array}\right] \\
G_{f i} & \triangleq\left[\begin{array}{llll}
C A^{i-2} K & \cdots & C K & I
\end{array}\right] \\
& \triangleq\left[\begin{array}{llll}
G_{i-1} & \cdots & G_{1} & G_{0}
\end{array}\right]
\end{aligned}
$$

$$
\forall i=1,2, \cdots, f
$$

where $H_{i}$ and $G_{i}$ are the Markov parameters for the deterministic input and innovation sequence, respectively. We have the following partitioned equations:

$$
\begin{gathered}
Y_{f i}=\Gamma_{f i} X_{k}+H_{f i} U_{i}+G_{f i} E_{i} \\
\forall i=1,2, \cdots, f
\end{gathered}
$$

Note that each of the above equation is guaranteed causal.

\subsection{Parallel Estimation of $\Gamma_{f i}$ and $H_{f i}$}

By eliminating $e(k)$ in the innovation model through iteration, it is straightforward to derive the following relation (Knudsen, 2001),

$$
X_{k}=L_{z} Z_{p}+A_{K}^{p} X_{k-p}
$$

where

$$
\begin{aligned}
L_{z} & \triangleq\left[\begin{array}{ll}
\Delta_{p}\left(A_{K}, K\right) & \Delta_{p}\left(A_{K}, B_{K}\right)
\end{array}\right](14 \mathrm{a}) \\
\Delta_{p}(A, B) & \triangleq\left[\begin{array}{lll}
A^{p-1} B \cdots & A B \quad B
\end{array}\right] \\
A_{K} & \triangleq A-K C \\
B_{K} & \triangleq B-K D
\end{aligned}
$$

Substituting this equation into Eq. 12, we obtain

$$
\begin{gathered}
Y_{f i}=\Gamma_{f i} L_{z} Z_{p}+\Gamma_{f i} A_{K}^{p} X_{k-p}+H_{f i} U_{i}+G_{f i} E_{i} \\
\forall i=1,2, \cdots, f
\end{gathered}
$$

Since the second term in the RHS of Eq. 15 tends to zero as $p$ tends to infinity, we have the following least squares estimates:

$$
\begin{aligned}
& {\left[\begin{array}{ll}
\hat{\Gamma}_{f i} L_{z} & \hat{H}_{f i}
\end{array}\right]=Y_{f i}\left[\begin{array}{c}
Z_{p} \\
U_{i}
\end{array}\right]^{+}} \\
& \forall i=1,2, \cdots, f
\end{aligned}
$$

Qin and Ljung (Qin and Ljung, 2003) point out that the PARSIM-P algorithm requires that the input $u(k)$ and innovation sequence $e(k)$ are uncorrelated, i.e., 
$\frac{1}{N} E_{i} U_{i}^{T} \rightarrow 0$ as $N \rightarrow \infty$, to be unbiased. Because of this requirement, the PARSIM-P algorithm is biased for closed-loop identification. In the next section we propose a new PARSIM algorithm, PARSIM-E, that estimates the past innovation process first. The estimated innovation is treated as known data and the subsequent projections do not require future input to be uncorrelated with past innovation, hence the PARSIM-E method is applicable to closed-loop identification.

\section{PARSIM WITH INNOVATION ESTIMATION}

By ignoring the second term on the RHS of Eq. 15 and setting $i=1$, we have

$$
Y_{f 1}=\left[\begin{array}{ll}
\Gamma_{f 1} L_{z} & H_{f 1}
\end{array}\right]\left[\begin{array}{c}
Z_{p} \\
U_{1}
\end{array}\right]+E_{1}
$$

Therefore, a least squares estimate of the innovation process is:

$$
\hat{E}_{1}=Y_{f 1}-\left[\begin{array}{ll}
\hat{\Gamma}_{f 1} L_{z} & \hat{H}_{f 1}
\end{array}\right]\left[\begin{array}{c}
Z_{p} \\
U_{1}
\end{array}\right]
$$

Now return to Eq. 15 for a general $i=2,3, \ldots, f$. Noticing that

$$
E_{i}=\left[\begin{array}{c}
E_{f 1} \\
E_{f 2} \\
\vdots \\
E_{f i}
\end{array}\right]=\left[\begin{array}{c}
E_{i-1} \\
E_{f i}
\end{array}\right]
$$

and replacing $E_{i-1}$ with $\hat{E}_{i-1}$, Eq. 15 becomes,

$$
Y_{f 1}=\left[\begin{array}{lll}
\Gamma_{f i} L_{z} & H_{f i} & G_{f i}^{-}
\end{array}\right]\left[\begin{array}{c}
Z_{p} \\
U_{i} \\
\hat{E}_{i-1}
\end{array}\right]+E_{f i}
$$

where

$$
G_{f i}^{-}=\left[C A^{i-2} K C A^{i-3} K \ldots C K\right] .
$$

The least squares estimate

$$
\left[\begin{array}{lll}
\hat{\Gamma}_{f i} L_{z} & \hat{H}_{f i} & \hat{G}_{f i}^{-}
\end{array}\right]=Y_{f 1}\left[\begin{array}{c}
Z_{p} \\
U_{i} \\
\hat{E}_{i-1}
\end{array}\right]^{+}
$$

now does not require future input $u_{k}$ to be uncorrelated with past innovation $e_{k}$. It only requires that future innovation to be independent of past input, which is always true for both open-loop and closed-loop data. The innovation data are calculated recursively using

$$
\hat{E}_{i}=\left[\begin{array}{c}
\hat{E}_{i-1} \\
\hat{E}_{f i}
\end{array}\right]
$$

With the least squares estimates from Eq. 22, the system matrices A, B, C, D, K can be estimated similarly to the procedures given in (Qin and Ljung, 2003).

\section{SIMULATION RESULTS}

We simulate the following process

$$
y_{k}+a y_{k-1}=b u_{k-1}+e_{k}+c e_{k-1}
$$

with a feedback controller

$$
u_{k}=-K y_{k}+r_{k}
$$

where $a=-0.9, b=1$, and $c=0.9$. The standard deviation for $e_{k}$ is one and that for $r_{k}$ is two; both of the signals are Gaussian white noise. Openloop experiments are simulated with $K=0$ and closed-loop experiments with $K=0.6$. In both cases 2000 data points are collected and 20 MonteCarlo simulations are performed. Figure 1 shows the pole estimates from PARSIM-E, PARSIM$\mathrm{P}$ and MOESP for open-loop and closed-loop data. There is no observed difference for open-loop identification, while the closed-loop identification results are very different. The PARSIM-E gives the best estimate without bias.

Figure 2 shows the box plots of the parameter estimates from 20 simulations. In the openloop case, all methods estimate a equally well. PARSIM-E and PARSIM-P give much better estimates for $b$ than MOESP, showing the benefit of parsimonious formulation. PARSIM-E and PARSIM-P give equally good estimates for $c$, while MOESP does not estimate the stochastic parameters. In the closed-loop case, the PARSIM$\mathrm{E}$ algorithm gives unbiased estimates for $a$ and $b$. Both PARSIM-P and MOESP fail on closedloop identification, with MOESP giving the worst results.

To examine the frequency responses of the identified models, Figure 3 gives the Bode plots by averaging the 20 closed-loop experiments. It is clearly shown that MOESP and PARSIM-P method fail to identify the steady state gain, while the PARSIM-E method is unbiased in all frequencies.

\section{CONCLUSIONS}

The proposed new subspace identification method with parsimonious models and innovation estimation gives unbiased results for closed-loop identification. For open-loop data both PARSIM-E and PARSIM-P algorithms give superior results than the contentional subspace model formulation. 

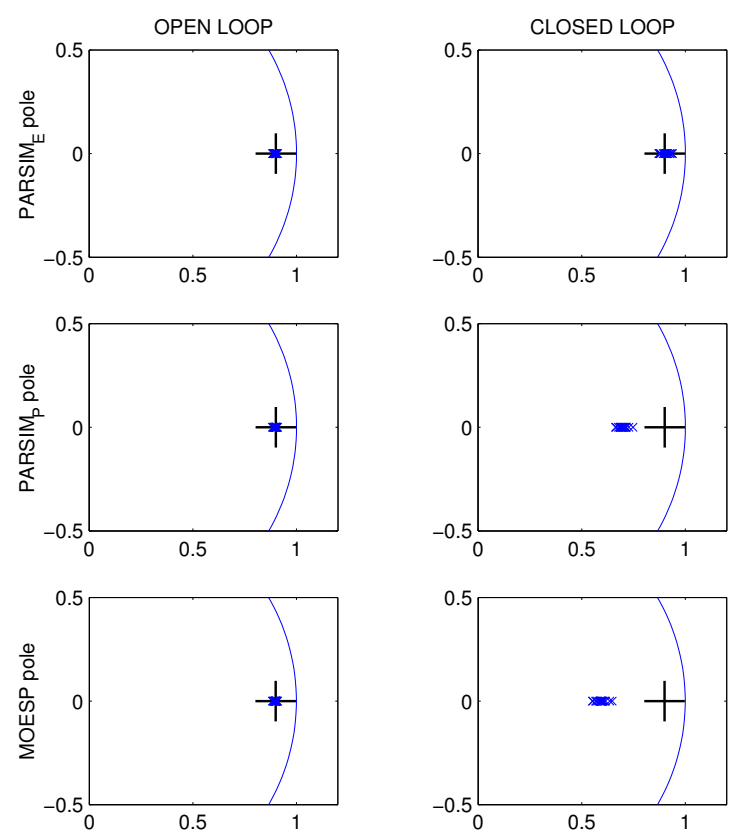

Fig. 1. Pole estimates for the simulation example.
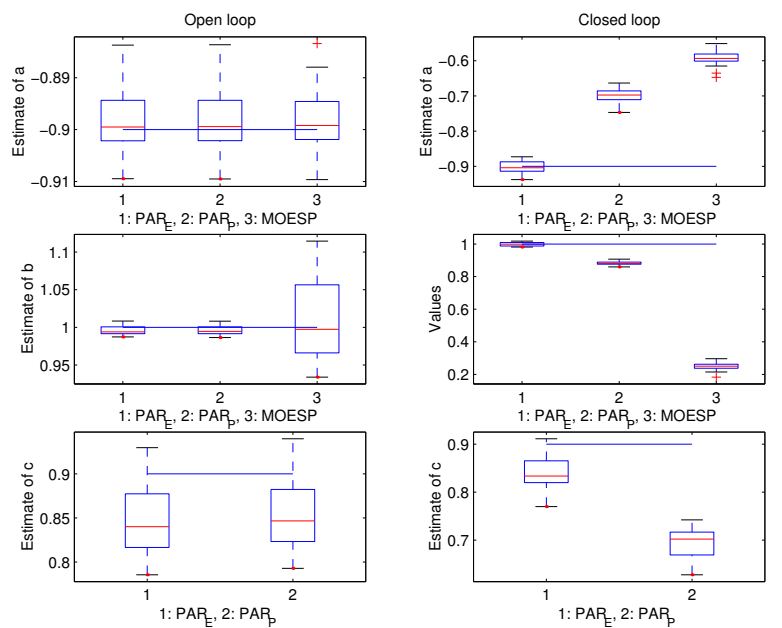

Fig. 2. Parameter estimates for the simulation example.

\section{ACKNOWLEDGMENTS}

Financial support from National Science Foundation under CTS-9985074 and a Faculty Research Assignment grant from University of Texas is gratefully acknowledged.

\section{REFERENCES}

Bauer, D., M. Deistler and W. Scherrer (1999). Consistency and asymptotic normality of some subspace algorithms for systems without observed inputs. Automatica 35, 12431254 .

Chou, C.T. and Michel Verhaegen (1997). Subspace algorithms for the identification of multivariable dynamic errors-in-variables models. Automatica 33(10), 1857-1869.
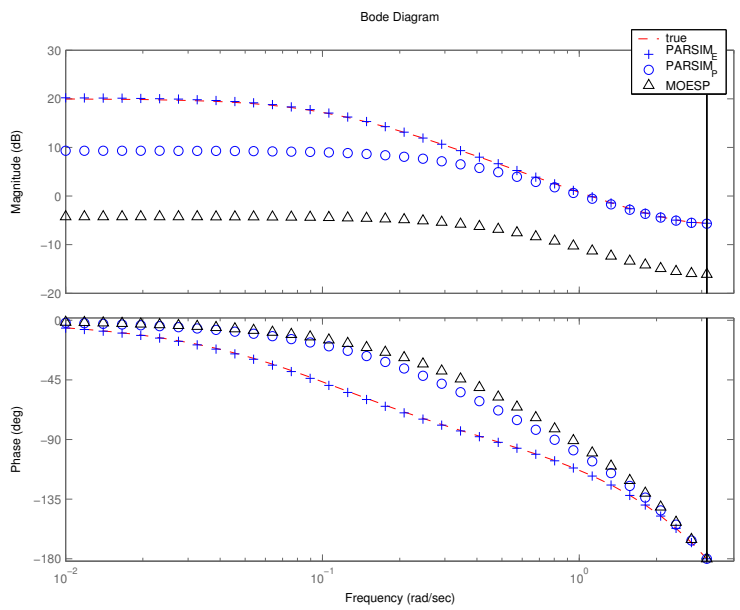

Fig. 3. Bode diagram for the closed-loop identification results

Deistler, M., K. Peternell and W. Scherrer (1995). Consistency and relative efficiency of subspace methods. Automatica 31, 1865-1875.

Gustafsson, Tony (2002). Subspace-based system identification: weighting and pre-filtering of instruments. Automatica 38, 433-443.

Heij, C. and W. Scherrer (1999). Consistency of system identification by global total least squares. Automatica 35, 993-1008.

Jansson, Magnus and Bo Wahlberg (1996). A linear regression approach to state-space subspace system. Signal Processing 52, 103-129.

Jansson, Magnus and Bo Wahlberg (1998). On consistency of subspace methods for system identification. Automatica 34(12), 1507-1519.

Knudsen, Torben (2001). Consistency analysis of subspace identification methods based on a linear regression approach. Automatica 37, 81-89.

Larimore, Wallace. E. (1983). System identification, reduced-order filtering and modeling via canonical variate analysis. In: Proceedings of the 1983 American Control Conference. pp. 445-451.

Larimore, Wallace. E. (1990). Canonical variate analysis in identification, filtering and adaptive control. In: Proceedings of the 29th Conference on Decision and Control. pp. 596-604.

Ljung, L. (1999). System Identification: Theory for the User. Prentice-Hall, Inc.. Englewood Cliffs, New Jersey.

Moonen, M., B. DeMoor, L. Vandenberghe and J. Vandewalle (1989). On and off-line identification of linear state-space models. International Journal of Control 49, 219-232.

Moor, B. De, J. Vandewalle, L. Vandenberghe and P. Van Mieghem (1988). A geometrical strategy for the identification of state space models of linear multivariable systems with singular value decomposition. In: Proceedings of the 8th IFAC Symp. on Identification and System Parameter Estimation. pp. 700-704. 
Overschee, P. Van and B. De Moor (1993). Subspace algorithms for the stochastic identification problem. Automatica 29, 649-660.

Overschee, P. Van and B. De Moor (1994). N4SID: Subspace algorithms for the identification of combined deterministic-stochastic systems. Automatica 30(1), 75.

Overschee, Peter Van and Bart de Moor (1995). A unifying theorem for three subspace system identification algorithms. Automatica 31(12), 1853-1864.

Overschee, Peter Van and Bart De Moor (1996). Subspace Identification for Linear Systems. Kluwer Academic Publishers.

Qin, S. J. and L. Ljung (2003). Parallel qr implementation of subspace identification with parsimonious models. In: IFAC Symposium on System Identification.

Verhaegen, M. (1991). A novel non-iterative mimo state space model identification techniques. In: Proceedings of the 9th IFAC/IFORS Symp. on Identification and System Parameter Estimation. pp. 1453-1458.

Verhaegen, M. and P. Dewilde (1992). Subspace model identification. part i: the output-error state-space model identification class of algorithms. International Journal of Control 56, 1187-1210.

Verhaegen, Michel (1994). Identification of the deterministic part of MIMO state space models given in innovations form from input-output data. Automatica 30(1), 61-74.

Viberg, M. (1994). Subspace methods in system identification. In: Proceedings of the 10th IFAC Symp. on Identification and System Parameter Estimation. pp. 1-12.

Viberg, Mats (1995). Subspace-based methods for the identification of linear time-invariant systems. Automatica 31(12), 1835-1851. 
Abstract

Keywords: 


Avdelning, Institution
Division, Department
Division of Automatic Control
Department of Electrical Engineering

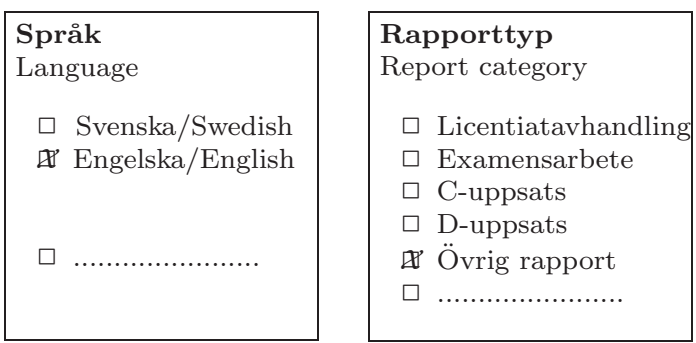

URL för elektronisk version

http://www. control.isy.liu.se

\begin{tabular}{|c|c|}
\hline ISBN & \\
\hline ISRN & \\
\hline $\begin{array}{l}\text { Serietitel och serienummer } \\
\text { Title of series, numbering }\end{array}$ & ISSN \\
\hline LiTH-ISY-R-2559 & \\
\hline
\end{tabular}

Titel

Title

Closed-loop Subspace Identification with Innovation Estimation

Författare

Author

S. Joe Qin, Lennart Ljung,

\section{Sammanfattning \\ Abstract}

Nyckelord

Keywords 